\title{
DESIGN \& DIFERENCIAL SEMÂNTICO: avaliação da percepção visual de grupos acadêmicos distintos através da análise de componentes principais
}

\author{
Rodrigo Holdschip \\ PPGDesign, Fac. De Arquitetura, Artes e Comunicação, UNESP \\ rodrigoholdschip@yahoo.com.br \\ João Fernando Marar \\ Departamento de Computação, Faculdade de Ciências, UNESP \\ fermarar@fc.unesp.br \\ Fabrício José Aguiar de Mira \\ Lab. de Sistemas Adaptativos e Computação Inteligente, UNESP \\ fabriciomira@gmail.com
}

\begin{abstract}
Resumo: O presente estudo investigou a influência da formação acadêmica sobre a percepção visual de usuários. Os dados utilizados neste estudo foram provenientes da aplicação da técnica do diferencial semântico utilizada para quantificar as respostas subjetivas dos participantes. 40 estudantes de graduação ( 20 alunos de design e 20 alunos de outras áreas) foram instruídos a avaliar 7 cadeiras e 3 poltronas. Análises por componentes principais foram realizadas a fim de reduzir a quantidade de informação inicial obtida e facilitar sua interpretação. Três fatores semânticos foram identificados: aparência, conforto e estilo. Os resultados também sugerem que a percepção dos usuários foi influenciada pela formação acadêmica.
\end{abstract}

Palavras-chave: design, usuário, produto, percepção, diferencial semântico.

\begin{abstract}
This study investigated the influence of academic training on visual perception of users. The data used in this study was derived from the application of the technique of semantic differential used to quantify the subjectives responses of the participants. 40 undergraduate students (20 students of design and 20 students from other areas) were instructed to evaluate 7 chairs and 3 armchairs. Principal components analyzes were performed in order to reduce the amount of initial information obtained and facilitate their interpretation. Three semantic factors were identified: appearance, comfort and style. The results also suggest that the perception of users were influenced by academic training.
\end{abstract}

Keywords: design, user, product, perception, semantic differential. 


\section{INTRODUÇÃO}

Após quase meio século sob a influência do funcionalismo - movimento racionalista surgido na Escola Superior de Design de Ulm, na Alemanha - cujo princípio estético era focado no objeto (a forma segue a função), o discurso do design pósmoderno se volta para o usuário e para a dimensão semântica do produto, onde a forma segue o significado (KRIPPENDORFF, 1995). As emoções provocadas por produtos tornaram-se um diferencial competitivo e vantajoso, pois recentes pesquisas indicam que elas desempenham um importante papel influenciando consumidores no momento da compra (DESMET, 2005).

O significado das emoções e experiências dos usuários para o domínio do design tem levado à introdução de várias técnicas e ferramentas objetivando a obtenção de informações sobre as interações subjetivas de usuários com produtos (BONAPACE 2002; DEMIR 2008). A relação emocional entre usuários e produtos é determinada em grande parte pela dimensão simbólica. Mesmo produtos práticos, tais como utensílios de cozinha, terão algum significado. Na escolha de tais produtos, consumidores tendem a ser influenciados por mensagens que eles acreditam ser transmitidas por estes, na medida em que sua impressão geral do produto corresponda à imagem que eles têm de si mesmos (MCDONAGH et al., 2002).

Diante disso, ressalta-se a importância do levantamento e interpretação dos dados referentes à percepção de usuários durante o processo de concepção de novos produtos, uma vez que, os significados atribuídos pelo designer às formas que ele cria não necessariamente serão percebidos da mesma maneira pelos usuários (KRIPPENDORFF, 1995).

Com o intuito de investigar a influência da formação acadêmica sobre a percepção visual de usuários em direção a produtos, o presente estudo explorou o uso da técnica do diferencial semântico (OSGOOD, 1957), um dos procedimentos mais utilizados, pois possibilita a quantificação da intensidade e do conteúdo das impressões subjetivas mais sutis e difíceis de descrever.

Os dados obtidos foram então submetidos a análises de componentes principais, uma técnica estatística multivariada voltada a explicar a estrutura de variância-covariância de um conjunto de variáveis através de combinações lineares, objetivando a redução da informação original (JOHNSON; WICHERN, 2007), facilitando sua interpretação e revelando relacionamentos que não foram previamente identificados (RENCHER, 2002).

\section{MATERIAIS E MÉTODOS}

A fim de investigar a influência da formação acadêmica sobre a percepção de usuários, um experimento foi elaborado para o caso particular de cadeiras e poltronas.

Dados foram coletados por meio de opiniões subjetivas obtidas a partir da aplicação da técnica do diferencial semântico (OSGOOD, 1957). Sete cadeiras e três poltronas representativas foram selecionadas e quarenta estudantes divididos em dois grupos com diferentes áreas de formação acadêmica participaram deste estudo. Nas subseções seguintes, é mostrada uma descrição detalhada dos materiais e métodos utilizados. 


\subsection{Composição da escala de diferencial semântico}

Usuários comunicam suas percepções através de palavras que possam expressar suas impressões que surgem ao interagir com produtos. Estas palavras formam o universo semântico do produto.

A escala de diferencial semântico utilizada neste estudo foi composta por doze pares de adjetivos bipolares considerados apropriados para a caracterização de cadeiras e poltronas: bonita - feia; confortável - desconfortável; delicada - grosseira; elegante - deselegante; espaçosa - compacta; estável - instável; extravagante discreta; feminina - masculina; decorativa - prática; inovadora - tradicional; dura macia; resistente - frágil. Estes adjetivos foram selecionados a partir de uma lista inicial, obtida através de textos contidos em materiais de divulgação impresso e online, após a eliminação dos adjetivos com significados equivalentes.

\subsection{Seleção das cadeiras e poltronas}

Para este estudo, interessado na investigação de respostas subjetivas de dois diferentes grupos de usuários em direção a produtos, sete cadeiras e três poltronas (Figura 1) com diferentes características de forma, cor, tamanho, aparência e materiais foram selecionadas.
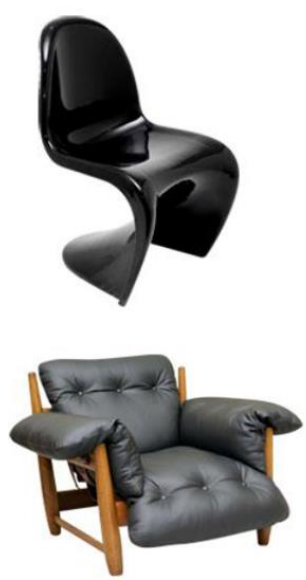
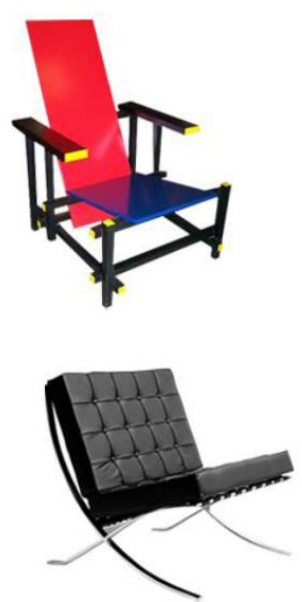
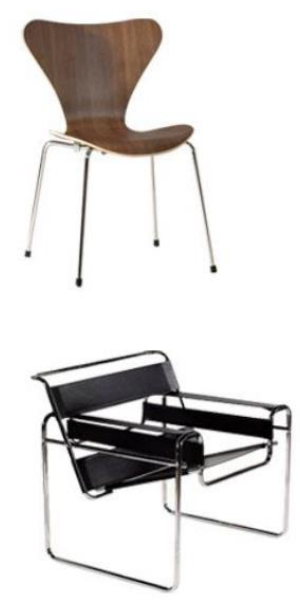
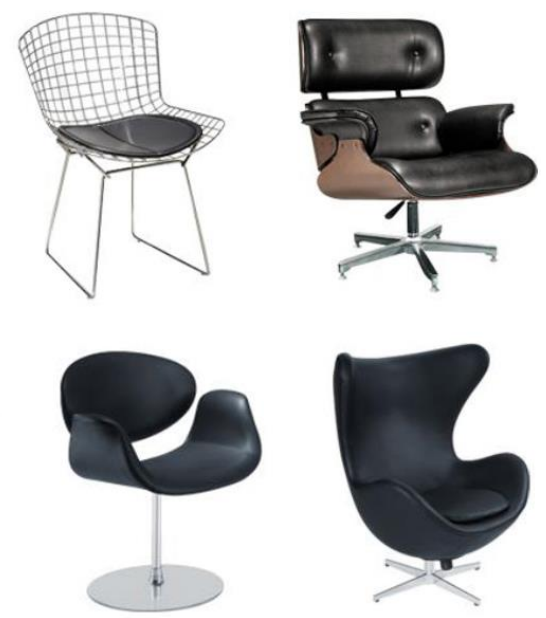

Figura 1 - Cadeiras e poltronas selecionadas para este estudo. Fonte: Fiell (2005).

\subsection{Seleção e caracterização dos participantes}

Quarenta alunos de graduação divididos em dois grupos de vinte estudantes cada participaram voluntariamente deste estudo: 1) estudantes de graduação em design (ED); 2) estudantes de graduação em outros cursos (EO). A Tabela 1 apresenta um resumo das principais características dos participantes.

Tabela 1 - Principais características dos 40 participantes deste estudo.

\begin{tabular}{llll}
\hline & ED & EO & Total \\
\hline Idade: Média (Desvio Padrão) & $27,4(10,2)$ & $26,2(8,0)$ & $26,8(9,1)$ \\
\hline Homens/Mulheres & $9 / 11$ & $9 / 11$ & $18 / 22$ \\
\hline
\end{tabular}

Fonte: Elaborado pelo autor, com base na pesquisa realizada. 


\subsection{Procedimento}

Através de um formulário elaborado e disponibilizado online, os quarenta participantes foram instruídos a avaliar as amostras (imagens) de cadeiras e poltronas (Figura 1) por meio da escala de diferencial semântico (Tabela 2). Para evitar préjulgamentos, as escalas foram espelhadas aleatoriamente e somente valores positivos foram atribuídos a elas, sendo numeradas de 0 a 3 para cada um dos descritores opostos pertencentes ao mesmo par.

Tabela 2 - Escala de diferencial semântico.

\begin{tabular}{l|r|r|r|r|r|r|r|r}
\hline & 3 & 2 & 1 & 0 & 1 & 2 & 3 & \\
\hline bonita & & & & & & & & feia \\
\hline confortável & & & & & & & & desconfortável \\
\hline delicada & & & & & & & & grosseira \\
\hline elegante & & & & & & & & deselegante \\
\hline espaçosa & & & & & & & & compacta \\
\hline estável & & & & & & & & instável \\
\hline extravagante & & & & & & & & discreta \\
\hline feminina & & & & & & & & masculina \\
\hline decorativa & & & & & & & & prática \\
\hline inovadora & & & & & & & & tradicional \\
\hline dura & & & & & & & & macia \\
\hline resistente & & & & & & & & frágil \\
\hline
\end{tabular}

Fonte: Elaborado pelo autor, com base na pesquisa realizada.

\subsection{Análises e discussão dos resultados}

Os dados obtidos para este estudo foram analisados em duas etapas que serão descritas a seguir: análise 1 e análise 2. Para realização de ambas as análises foi utilizado o aplicativo SPSS Statistics R17.0.

\subsubsection{Análise 1: identificação do espaço semântico de cadeiras e poltronas}

A fim de reduzir a quantidade de informação inicial obtida por este estudo e facilitar sua interpretação, os dados coletados a partir das 400 avaliações (40 participantes $X 10$ cadeiras e poltronas) realizadas por ambos os grupos foram submetidos a uma Análise de Componentes Principais (PCA) inicial através da utilização do aplicativo SPSS Statistics R17.0, com método de rotação ortogonal dos eixos Varimax, com o intuito de encontrar a rotação dos fatores que maximizasse a variância da matriz de pesos, contribuindo para a simplificação da interpretação dos fatores (BOUROCHE; SAPORTA, 1980).

A fim de garantir a produção de fatores representativos através da PCA inicial, variáveis que apresentaram medidas de adequação da amostra (MSA) e comunalidades inferiores a 0,5 foram eliminadas (HAIR et al., 2009) durante a execução de análises preliminares, resultando na especificação de um conjunto de dez variáveis suficientemente correlacionadas: bonita - feia; confortável - desconfortável; delicada grosseira; elegante - deselegante; espaçosa - compacta; extravagante - discreta; feminina - masculina; decorativa - prática; inovadora - tradicional; dura - macia. 
O resultado da PCA inicial revela que os dez pares de adjetivos opostos utilizados para configurar o espaço semântico dos produtos avaliados podem ser classificados em três fatores principais com 72,405\% de variância explicada (Tabela 3). Entende-se por fator, o conjunto de variáveis altamente correlacionadas combinadas linearmente, com objetivo de resumir as informações provenientes das diversas variáveis. Para determinar o número de fatores foi utilizado como base o critério de Kaiser, que considera como mais significativos os autovalores superiores a 1 (JOHNSON; WICHERN, 2007).

Tabela 3 - Matriz de Componentes Rotacionada.

\begin{tabular}{l|c|c|c}
\hline \multirow{2}{*}{} & \multicolumn{3}{|c}{ Componentes } \\
\cline { 2 - 4 } & $\mathbf{1}$ & $\mathbf{2}$ & $\mathbf{3}$ \\
\hline Delicada-Grosseira &, 876 & & \\
\hline Elegante-Deselegante &, 817 & & \\
\hline Bonita-Feia &, 771 & & \\
\hline Feminina-Masculina &,- 576 & & \\
\hline Dura-Macia & &,- 860 & \\
\hline Confortável-Desconfortável & &, 839 & \\
\hline Espaçosa-Compacta & &, 824 & \\
\hline Funcional-Estética & & &, 808 \\
\hline Extravagante-Discreta & & &, 801 \\
\hline Inovadora-Tradicional & & &, 778 \\
\hline \multicolumn{1}{c}{ \% de Variância Explicada } & 26,444 & 25,205 & 20,757 \\
\hline \multicolumn{1}{c}{ Autovalor } & 26,444 & 51,649 & 72,405 \\
\hline
\end{tabular}

Fonte: Elaborado pelo autor, com base na pesquisa realizada.

Com o intuito de verificar a adequação da PCA, recorreu-se ao teste de esfericidade de Barlett e à Medida de Adequação da Amostra (MSA). O teste de esfericidade de Barlett avaliou a PCA como sendo estatisticamente significante $($ sign. $=0,00)$ indicando a existência de correlações suficientes entre as variáveis envolvidas e uma MSA de 0,748 foi obtida, indicando que o grau de intercorrelação entre as variáveis foi mediano e, portanto aceitável (HAIR et al. 2009).

Para interpretação dos fatores extraídos foram levadas em consideração somente as variáveis com cargas fatoriais acima de 0,5 (HAIR et al., 2009). Os três fatores semânticos podem ser interpretados e nomeados da seguinte forma:

Fator 1: Aparência. Este primeiro fator, que responde por 26,444\% da variância explicada, reúne características relacionadas à aparência dos produtos (delicadagrosseira, elegante-deselegante, bonita-feia e feminina-masculina).

Fator 2: Conforto. Este segundo fator, que responde por 25,205\% da variância explicada, reúne características de cadeiras e poltronas relacionadas ao conforto (dura-macia, confortável-desconfortável e espaçosa-compacta). 
Fator 3: Estilo. Este terceiro fator que responde por 20,757\% da variância explicada, reúne características relacionadas ao estilo dos produtos (funcional-estética, extravagante-discreta e inovadora-tradicional).

\subsubsection{Análise 2: estabilidade dos fatores semânticos entre os grupos de participantes}

A fim de comparar os agrupamentos dos fatores semânticos de acordo com os dois grupos de participantes ED e EO, foram realizadas duas PCAs independentes com método de rotação ortogonal dos eixos Varimax a partir dos dados coletados durante a avaliação dos produtos selecionados para este estudo. Para as PCAs, foi utilizado o mesmo conjunto constituído pelas 10 variáveis suficientemente correlacionadas utilizadas na PCA inicial.

Novamente, com o intuito de se verificar a adequação das PCAs independentes, recorreu-se ao teste de esfericidade de Barlett e à Medida de Adequação da Amostra (MSA). Em ambas as PCAs o teste de esfericidade de Barlett indicou a existência de correlações suficientes entre as variáveis envolvidas nas análises $($ sign. $=0,00)$ e foram obtidas MSAs superiores a 0,732, indicando que o grau de intercorrelação entre as variáveis foi aceitável. Todas as variáveis envolvidas nas duas análises obtiveram MSAs individuais superiores a 0,619 e comunalidades superiores a 0,5.

Os resultados das análises fatoriais independentes revelam que em ambas as análises os dez pares de adjetivos opostos utilizados para configurar o espaço semântico em relação aos produtos avaliados podem ser classificados em três fatores principais com variância explicada superiores a 71,715\% e autovalores superiores a 1 .

A tabela 4 fornece uma representação gráfica da distribuição das dez variáveis na composição dos fatores (F1, F2 e F3) obtidos através das três PCAs realizadas. Por uma questão de simplificação, as cargas fatoriais de cada variável foram omitidas e os dez pares de adjetivos foram agrupados por "conceitos".

Tabela 4 - Composição dos fatores por PCA independente.

\begin{tabular}{|c|c|c|c|c|c|c|c|c|c|c|}
\hline \multirow{2}{*}{ Conceito } & \multirow{2}{*}{ Variáveis } & \multicolumn{3}{|c|}{ Geral } & \multicolumn{3}{|c|}{ ED } & \multicolumn{3}{|c|}{ EO } \\
\hline & & F1 & F2 & F3 & F1 & F2 & F3 & F1 & $\mathbf{F} 2$ & F3 \\
\hline \multirow{4}{*}{ Aparência } & Delicada-Grosseira & $\bullet$ & & & $\bullet$ & & & & $\bullet$ & \\
\hline & Elegante-Deselegante & $\bullet$ & & & $\bullet$ & & & & $\bullet$ & \\
\hline & Bonita-Feia & $\bullet$ & & & $\bullet$ & & & & $\bullet$ & \\
\hline & Feminina-Masculina & $\bullet$ & & & $\bullet$ & & & & $\bullet$ & \\
\hline \multirow{3}{*}{ Conforto } & Dura-Macia & & $\bullet$ & & & $\bullet$ & & $\bullet$ & & \\
\hline & Confortável-Desconfortável & & $\bullet$ & & & $\bullet$ & & $\bullet$ & & \\
\hline & Espaçosa-Compacta & & $\bullet$ & & & $\bullet$ & & $\bullet$ & & \\
\hline \multirow{3}{*}{ Estilo } & Funcional-Estética & & & $\bullet$ & & & $\bullet$ & & & $\bullet$ \\
\hline & Extravagante-Discreta & & & $\bullet$ & & & $\bullet$ & & & $\bullet$ \\
\hline & Inovadora-Tradicional & & & - & & & - & & & - \\
\hline
\end{tabular}

Fonte: Elaborado pelo autor, com base na pesquisa realizada. 
A partir da tabela 4 é possível observar que, diferentemente das demais PCAs, na PCA "EO", as características relacionadas ao conceito de aparência passaram a compor não mais o primeiro fator, mas sim o segundo, respondendo assim pela segunda maior parcela da variância explicada. Enquanto que, a maior parcela passou a ser de responsabilidade das características relacionadas ao conforto, que passaram a compor nesta PCA o primeiro fator.

Isto demostra que o primeiro grupo, composto por designers, se focalizou principalmente nos aspectos relacionados à aparência dos produtos durante a avaliação, enquanto que, o segundo grupo, composto por estudantes de outras áreas, se focalizou principalmente nos aspectos relacionados ao conforto do uso. Fato que se justifica mediante as diferenças existentes entre as formações acadêmicas recebidas por ambos os grupos.

Também é possível observar que, embora as ordens dos fatores tenham se alterado em função da formação acadêmica de cada grupo, a composição dos mesmos manteve-se estável.

\section{CONCLUSÃO}

A utilização da análise por componentes principais em um conjunto de dados, como os obtidos a partir da utilização da técnica de diferencial semântico, pode fornecer a simplificação dos dados originais objetivando uma melhor observação das variáveis analisadas.

Uma escala de diferencial semântico composta por 12 pares de adjetivos bipolares foi utilizada para quantificar as avaliações dos participantes em direção a cadeiras e poltronas utilizadas neste estudo. Uma análise de componentes principais inicial, aplicada a somente dez variáveis altamente correlacionadas, demonstra que três fatores semânticos foram percebidos com relação aos produtos avaliados neste estudo: aparência, conforto e estilo.

Os resultados das duas análises de componentes principais independentes realizadas a partir dos dados coletados de ambos os grupos, sugerem que a formação acadêmica dos estudantes influencia significativamente o processo de percepção visual das características relacionadas à aparência e conforto de cadeiras e poltronas.

Acredita-se que estas descobertas forneçam uma sistemática compreensão sobre a percepção de usuários em direção a cadeiras e poltronas, podendo ser aplicada a outras categorias de produtos.

\section{REFERÊNCIAS}

BOUROCHE, J. M.; SAPORTA, G. Análise de dados. Rio de Janeiro: Zahar, 1980.

BONAPACE, L. Linking Product Properties to Pleasure: the sensorial quality assesment method - SEQUAM. In: GREEN, W. S.; JORDAN, P. W. Pleasure with products: beyond usability. London: Taylor \& Francis, 2002.

DEMIR, E. The Field of Design and Emotions: Concepts, Arguments, Tools, And Current Issues. Metu Journal of The Faculty of Arquitecture, Marmaris, v. 25, n. 1, p. 135-152, 2008.

DESMET, P. M. A. Measuring Emotions: development and application of an instrument to measure emotional responses to products. In: BLYTHE, M. A.; OVERBEEKE, K.; 
MONK, A. F. (Eds). Funology: from usability to Enjoyment. Dordrecht: Kluwer Academic Publishers, 2005. Cap. 9, p. 111-123.

FIELL, P. 1000 Chairs. São Paulo: Taschen do Brasil, 2005.

HAIR, J. F. et al. Análise multivariada de dados. Tradução de Adonai Schlup Sant'Anna. 6. ed. Porto Alegre: Bookman, 2009. ISBN 978-85-7780-534-1.

JOHNSON, R. A.; WICHERN, D.W. Applied multivariate statistical analysis. 6. ed. New Jersey: Pearson Prentice-Hall, 2007.

KRIPPENDORFF, K. On the essential contexts of artifacts or on the proposition that 'design is making sense (of things)'. In: MARGOLIN, V.; BUCHANAN, R. The Idea of Design. 2a. ed. Cambridge: MIT Press, 1995. p. 156-184.

MCDONAGH, D.; BRUSEBERG, A.; HASLAM, C. Visual product evaluation: exploring users' emotional relationships with products. Applied Ergonomics, v. 33, n. 3, p. 231240, Maio 2002. ISSN ISSN 0003-6870.

OSGOOD, C. E. The Measurement of meaning. Illinois: University of Illinois Press, 1957.

RENCHER, A. C. Methods of multivariate analysis. 2. ed. New York: John Wiley and Sons, 2002. 